\title{
Salmonella spp. in patients suffering from enteric fever and food poisoning in Thamar city, Yemen
}

\author{
R.R. Taha, ${ }^{7}$ S.M. Alghalibi and M.G. Saeed Saleh ${ }^{7}$
}

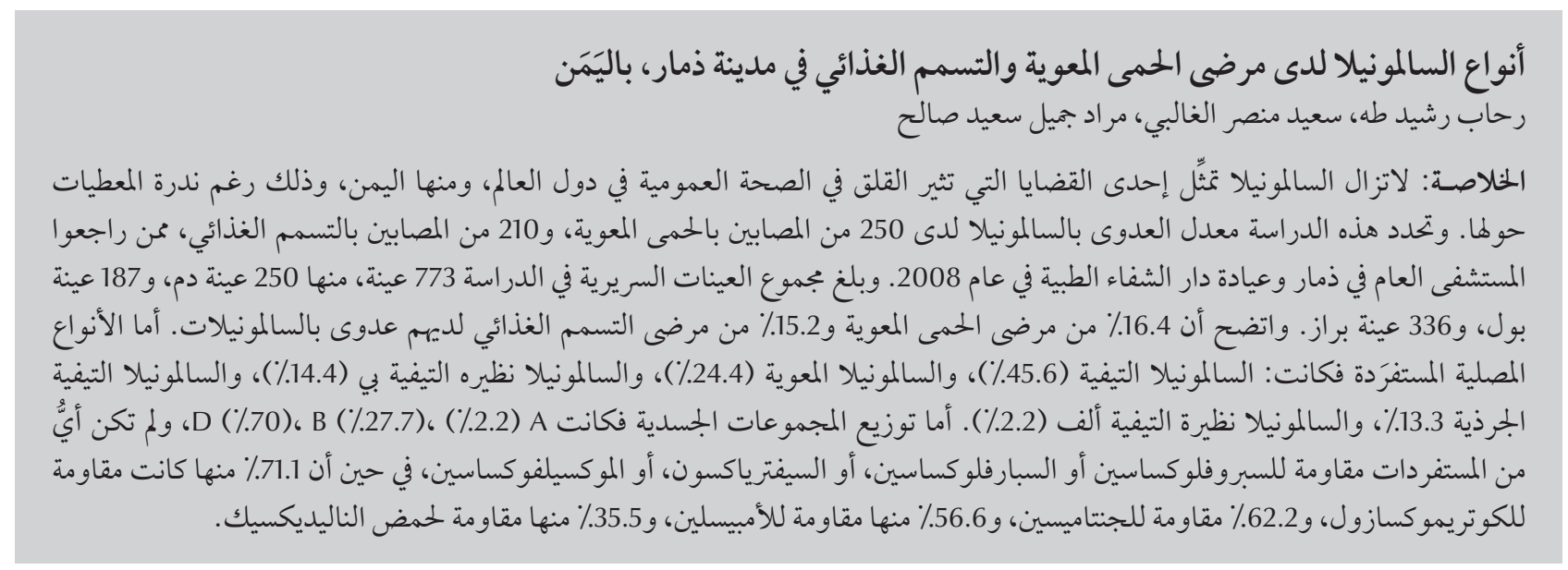

ABSTRACT Salmonella remains a public health concern around the world, including Yemen although data on its incidence are few. This study determined the incidence of Salmonella infection in 250 enteric fever and 210 food poisoning cases attending Thamar general hospital and Dar Alshafaa medical clinic in 2008. In total, 773 clinical specimens were taken: 250 blood, 187 urine and 336 stool samples. Of the patients with enteric fever and food poisoning, 16.4\% and 15.2\% respectively were infected with Salmonella. The serovars isolated were: Salmonella Typhi (45.6\%), Salmonella Enteritidis (24.4\%), Salmonella Paratyphi B (14.4\%), Salmonella Typhimurium (13.3\%) and Salmonella Paratyphi A (2.2\%). The distribution of somatic groups was: D (70\%), B (27.7\%) and A (2.2\%). None of the isolates was resistant to ciprofloxacin, sparfloxacin, ceftriaxone or moxifloxacin, while $71.1 \%$ were resistant to co-trimoxazole, $62.2 \%$ to gentamicin, $56.6 \%$ to ampicillin and $35.5 \%$ to nalidixic acid.

Infection à Salmonella spp. chez des patients atteints d'une fièvre entérique et d'une intoxication alimentaire dans la ville de Thamar (Yémen)

RÉSUMÉ Les salmonelles restent une préoccupation de santé publique dans le monde, y compris au Yémen, même si les données sur leur incidence sont rares. La présente étude a déterminé l'incidence de l'infection à Salmonella chez 250 cas de fièvre entérique et 210 cas d'intoxication alimentaire s'étant présentés à I'hôpital général de Thamar et dans l'établissement de soins de santé Dar Alshafaa en 2008. Au total, 773 échantillons cliniques ont été recueillis : 250 échantillons de sang, 187 échantillons d'urine et 336 échantillons de selles. Parmi les patients souffrant de fièvre entérique et d'intoxication alimentaire, 16,4\% et 15,2\% respectivement étaient infectés par une salmonelle. Les sérotypes isolés étaient les suivants : Salmonella Typhi (45,6 \%), Salmonella Enteritidis (24,4 \%), Salmonella Paratyphi B (14,4 \%), Salmonella Typhimurium (13,3\%) et Salmonella Paratyphi A (2,2\%). La répartition des groupes somatiques était la suivante : D (70 \%), B (27,7\%) et A (2,2\%). Aucun de ces isolats n'était résistant à la ciprofloxacine, à la sparfloxacine, à la ceftriaxone ou à la moxifloxacine, alors que 71,1\% étaient résistants au co-trimoxazole, 62,2 \% à la gentamicine, 56,6 \% à l'ampicilline et 35,5\% à l'acide nalidixique. 


\section{Introduction}

Salmonella Typhi and Salmonella Paratyphi are prevalent in developing countries causing frequent illness. However, they also a potential threat to the more developed nations due to increasing air travel and global operations. For example, enteric fever, which is caused by strains of Salmonella Typhi or Salmonella Paratyphi A, B or C, is estimated to occur in 16 million people a year causing 600000 deaths worldwide [1]. Non-typhoidal Salmonella (NTS) infections (salmonellosis) are one of the most important causative agents of acute diarrhoeal disease in children and adults. The World Health Organization has determined that NTSs are emerging as one of the most important etiological agents of infectious diseases in the world [2]. Over the last 2 decades the incidence NTS infections has increased considerably and reached epidemic levels in several countries [3]. Recently, specific serovars have been linked with certain foods or exposure. NTSs are primary pathogens of animals (e.g. poultry, cows, pigs, birds, sheep) and approximately $95 \%$ of human salmonellosis cases are associated with consumption of contaminated food such as meat, poultry, eggs, milk, seafood and fresh produce [4].

Antibiotics therapy is not recommended for uncomplicated Salmonella gastroenteritis. However, determination of antimicrobial resistance patterns is often valuable for surveillance purposes and may be performed periodically to monitor the development and spread of antibiotics resistance among these strains [5]. Resistance has emerged even to newer, more potent antimicrobial agents and the development of resistance to multiple drugs is a major problem in the treatment of salmonellosis [6]. Resistance to commonly used antibiotics, such as chloramphenicol, ampicillin and co-trimoxazole, has been reported from different parts of world in the last 2 decades [7].
In Yemen, Salmonella cases tend not to be recorded and so the incidence of Salmonella infection is not clearly known. In addition, the diagnosis procedure does not follow up-to-date methods used by international laboratories. Furthermore, local laboratory equipment and resources are limited and the number of trained personnel dealing with this issue is inadequate.

The aim of this study therefore was to determine the incidence of Salmonella cases in Thamar general hospital and Dar Alshafaa medical clinic, Thamar, Yemen. This research will add to the knowledge about the specific serotypes of Salmonella in Yemen that cause food poisoning and fever; this information has not been reported before and provides important in epidemiological data.

\section{Methods}

The study included patients with suspected enteric fever or food poisoning who were attending General Thamar hospital and Dar Alshafaa hospital in 2008 when the research team was present and from whom clinical specimens were taken.

Blood samples were inoculated in tryptic soy broth while stool and urine samples were inoculated in selenite and tetrathionate broth. They were then streaked on the differential media xylose lysine deoxycholate (XLD) and salmonella-shigella agar (SSA). All samples were incubated at $37^{\circ} \mathrm{C}$ for $24 \mathrm{~h}$. After incubation all colonies that gave typical characteristics of Salmonella were submitted for biochemical testing according to Holt et al. [8]. Finally, all presumptive Salmonella isolates were sent for serotyping at the Micro Analytical Centre in Cairo University, Egypt, according to the Kauffmann white scheme [9]. The difference between the 2 selective enrichment media in isolation of Salmonella serovars was examined using one-way ANOVA.
All confirmed Salmonella isolates were examined for antibiotic sensitivity using the Kirby-Bauer disk diffusion method (National Committee for Clinical Laboratory Standards).

\section{Results}

A total 460 patients who visited were included in this study: 250 with suspected enteric fever and 210 with suspected food poisoning. There were 249 females and 211 males, and their ages ranged between 5 and 60 years. From these patients, 773 clinical specimens were collected: 250 blood samples, 187 urine samples and 126 stool samples from patients with suspected enteric fever and 210 stool samples from patients with suspected food poisoning.

Of the 250 patients with suspected enteric fever, only 41 (16.4\%) were confirmed with Salmonella infection, while only $32(15.2 \%)$ of the 210 suspected food poisoning patients were infected with Salmonella.

The total number of confirmed Salmonella isolates obtained from the different clinical samples examined was 90: 58 isolates from patients with enteric fever and 32 from patients with food poisoning. These isolates comprised 5 serovars: Salmonella Typhi (41 isolates), Salmonella Enteritidis (22), Salmonella Paratyphi B (13), Salmonella Typhimurium (12) and Salmonella Paratyphi A (2) (Table 1). Salmonella Typhi, Salmonella Paratyphi A and Salmonella Paratyphi B were only found in people with enteric fever. Salmonella Typhimurium was only isolated from patients with food poisoning. Only Salmonella Enteritidis was isolated from both groups. The most prevalent serovar in enteric fever patients was Salmonella Typhi, which represented $70.7 \%$ of the total serovars isolated, followed by Salmonella Paratyphi B 22.4\% (Table 1). The most prevalent serovar in food poisoning was Salmonella Enteritidis which 


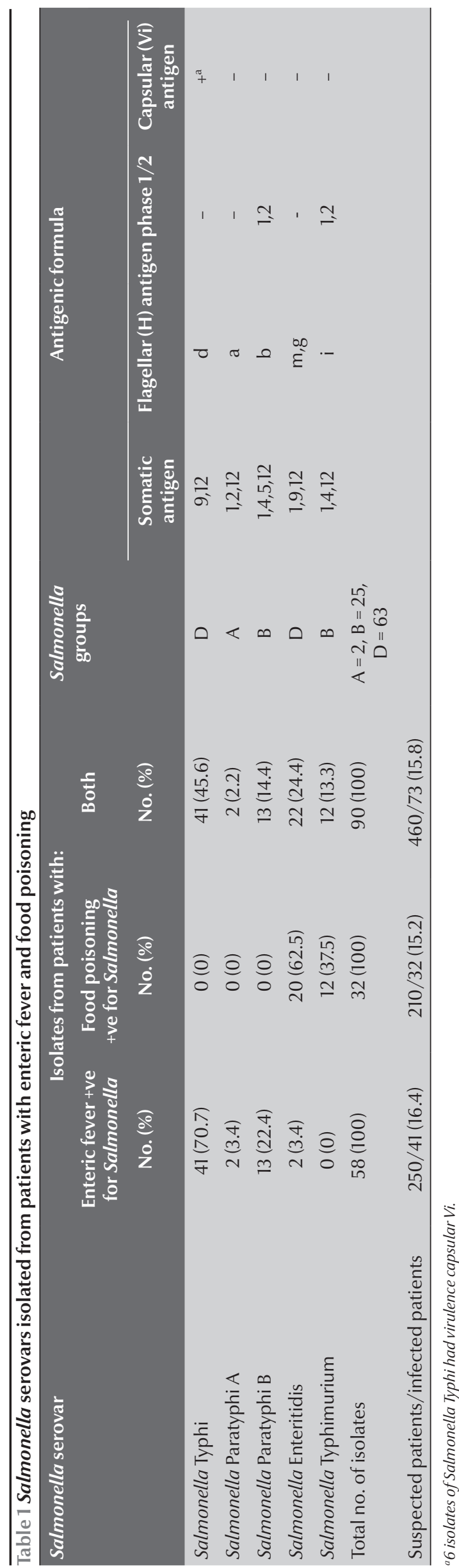

represented $62.5 \%$ of the total serovars, followed by Salmonella Typhimurium 37.5\% (Table 1).

The antigenic formula of the Salmonella serovars is also shown in Table 1. The serovars belonged to 3 somatic groups: group D, which represented $70 \%$, group B $27.7 \%$ and group A 2.2\%. The most prevalent serovar was Salmonella Typhi which represented 45.6\%, then Salmonella Enteritidis 24.4\%, Salmonella Paratyphi B 14.4\%, Salmonella Typhimurium 13.3\% and Salmonella Paratyphi A 2.2\% (Table 1).

Comparison of the selective enrichment media for isolation of Salmonella serovars showed tetrathionate broth to be more effective than selenite broth. With enrichment tetrathionate broth, Salmonella was isolated from 73 (14.0\%) stool and urine samples, compared to 45 (8.6\%) positive samples using selenite broth. Direct streaking or plating on differential agar media (XLD and SSA) only produced 22 (4.2\%) positive Salmonella samples (Table 2). There was a statistically significant difference between the 2 selective enrichment media $(P<0.01)$.

We evaluated the frequency infection with Salmonella according to age and sex (Table 3). Infection was more frequent in people aged 11-20 years (35.7\%) followed by those aged 21-30 years $(20.5 \%)$. As regards sex, infection was more common in women $(54.8 \%)$ than men.

Thirteen antibiotics were tested for sensitivity. The resistance pattern of the isolates is shown in Table 4. None of the isolates was resistant to ciprofloxacin, sparfloxacin, ceftriaxone and moxifloxacin. However, $71.1 \%$ were resistant to co-trimoxazole, $62.2 \%$ to gentamicin, $56.6 \%$ to ampicillin and $35.5 \%$ to nalidixic acid (Table 4).

\section{Discussion}

We found that of patients with suspected enteric fever or food poisoning, $16.4 \%$ and $15.2 \%$ respectively were confirmed with Salmonella infection.

For enteric fever, 4 serotypes were isolated, the most common being Salmonella Typhi (70.7\%) followed by Salmonella Paratyphi (25.8\%). The high prevalence of Salmonella Typhi may be due to the fact that Salmonella Typhi is spread predominantly within the household, whereas Salmonella Paratyphi is mainly transmitted outside the home [10]. Sources and sites of contamination of Salmonella include house members with clinical disease, pets with sub-clinical infection, contaminated items brought into the home, toilet bowls, carpet, floors, refrigerators and kitchen sinks and counter tops [10]. Consistent with this, independent risk factors for the intra-household spread of Salmonella Typhi are poor hand-washing hygiene and sharing of food from the same plate [11]. In Yemen, there are many popular dishes that are eaten by hand (without a spoon) and shared between family members, which can help the spread of 


\begin{tabular}{lcc}
\hline Table 2 Effect of selective medium on the isolation of Salmonella serovar \\
\hline Medium & Positive samples \\
& No. & $\%(\boldsymbol{n}=\mathbf{5 2 3})$ \\
Tetrathionate broth then streaked on XLD and SSA & 73 & 14 \\
Selenite broth then streaked on XLD and SSA & 45 & 8.6 \\
Plating directly on XLD and SSA & 22 & 4.2 \\
\hline
\end{tabular}

${ }^{a} 336$ stool 336 and 187 urine samples.

$X L D=x y l o s e$ lysine deoxycholate; SSA = salmonella-shigella agar .

Salmonella Typhi among members of one family or several families. On the other hand, risk factors for transmission of Salmonella Paratyphi tend to be outside the household (e.g. consumption of foods from street vendors). The low hygiene standards of street vendors contribute not only to the transmission of paratyphoid fever but also other foodborne diseases such as typhoid [11].

In our study both Salmonella Enteritidis (62.5\%) and Salmonella Typhimurium $(37.5 \%)$ were isolated from patients with food poisoning. This is to be expected because Salmonella Enteritidis accounts for approximately onefifth of all human Salmonella isolates reported to the Centers for Disease Control and Prevention (CDC), Atlanta, each year. Out of 371 outbreaks reported to CDC between 1985 and 1999, in which a vehicle of transmission was identified, $80 \%$ were egg-associated [12]. In a previous study in Thamar,

Salmonella Typhimurium, Salmonella Enteritidis, Salmonella Anatum, Salmonella Newport, Salmonella Arizona, and Salmonella Muenchen were isolated from poultry accessories, workers' hands and tools of the slaughter [13], indicating the potential risk to spread infection.

Another study in India found that Salmonella Typhi (55.5\%) followed by Salmonella Paratyphi A (44.5\%) were the most common isolates in enteric fever [14], while in Egypt, only Salmonella Typhi was isolated from patients with enteric fever [15]. In the Islamic Republic of Iran the most common isolate from patients with enteric fever was Salmonella Typhi (69.6\%), while in food poisoning Salmonella Typhimurium (69.0\%), was the most common serovar [16]. In non-typhoidal Salmonella food poisoning in Korea, Salmonella Enteritidis (68\%) and Salmonella Typhimurium $(32 \%)$ were the most common

\begin{tabular}{lcc}
\hline Table 3 Salmonella infection by of age and sex & \\
\hline Variable & $\begin{array}{c}\text { Patients positive for Salmonella } \\
\text { No. }\end{array}$ \\
Age group (years) & 11 & 14.5 \\
$5-10(n=76)$ & 35 & 35.7 \\
$11-20(n=98)$ & 16 & 20.5 \\
$21-30(n=78)$ & 3 & 6.3 \\
$31-40(n=47)$ & 3 & 3.1 \\
$41-50(n=96)$ & 5 & 7.7 \\
$51-60(n=65)$ & 73 & 15.8 \\
Total $(n=460)$ & & \\
Sex & 40 & 54.8 \\
Female $(n=249)$ & 33 & 45.2 \\
Male $(n=211)$ & 73 & 100 \\
Total $(n=460)$ & & \\
\hline
\end{tabular}

serovars, which is similar to the results of our study [17]. This difference between our study and these studies may be due to the difference in geographical region which is known to affect the prevalence of the different Salmonella serovars. In addition, difference in serotypes of Salmonella isolated between our results and those of other studies may be due to differences in trade, customs and food preparation methods.

In our study, the Salmonella isolates belonged to 3 groups, the most prevalent being group D (70\%). A previous study done in Yemen in 2001 reported that the majority of isolates in childhood diarrhoea cases were Salmonella group C followed by Salmonella group B [18]. This study was concerned with fever and diarrhoea caused by typhoid and paratyphoid strains while we, in addition to this, evaluated food poisoning and enteric fever strains caused by other strains of Salmonella, and this may account for the difference between their findings and ours. Also it is possible that new strains may enter to the country with feed, animals, food or foreigner people. Although Thamar and Sana'a cities are in a similar geographical area, they differ in the nature of their populations. Most of the inhabitants of Thamar city are from rural areas; they tend to be uneducated and their knowledge of public health is low. On the other hand, in Sana'a, being a capital city, the people are more urban and educated. A study in Saudi Arabia found that the majority of isolates were Salmonella group D $33 \%$, followed by group B 30\%, group C 24\%, Salmonella spp. $8 \%$ and group E 5\% [19].

Tetrathionate broth was more successful in isolation of isolates than selenite broth. These results are consistent with those obtained in Mexico, where out of 88 stool samples, $29.5 \%$ were positive with sodium tetrathionate, compared with only $7.9 \%$ with sodium selenite [20]. When clinical samples were streaked on XLD and SSA directly, isolation of Salmonella was very low. 


\begin{tabular}{|c|c|c|c|c|c|c|c|c|c|}
\hline \multirow[t]{3}{*}{ Salmonella serovar } & \multicolumn{9}{|c|}{ Antibiotic $^{\mathrm{a}}$} \\
\hline & C & NA & NX & AS & $\mathbf{S}$ & G & $\mathrm{CO}$ & CFX & AK \\
\hline & No (\%) & No $(\%)$ & No (\%) & No (\%) & No (\%) & No (\%) & No (\%) & No (\%) & No (\%) \\
\hline Salmonella Typhi $(n=41)$ & $6(14.6)$ & $15(41.6)$ & $1(2.4)$ & $17(41.5)$ & $1(2.4)$ & $22(53.7)$ & $23(56.1)$ & $0(0)$ & $6(14.6)$ \\
\hline Salmonella Enteritidis $(n=22)$ & $4(18.2)$ & $8(36.4)$ & $0(0)$ & $18(81)$ & $0(0)$ & $14(63.6)$ & $19(86.4)$ & $0(0)$ & $3(13.6)$ \\
\hline Salmonella Paratyphi B $(n=13)$ & $1(7.7)$ & $3(23)$ & $0(0)$ & $8(61.5)$ & $0(0)$ & $9(69.2)$ & $11(84.6)$ & $0(0)$ & $0(0)$ \\
\hline Salmonella Typhimurium $(n=12)$ & $1(8.3)$ & $5(41.7)$ & $0(0)$ & $7(58.3)$ & $0(0)$ & $10(83.3)$ & $9(75)$ & $2(16.6)$ & $1(8.3)$ \\
\hline Salmonella Paratyphi A $(n=2)$ & $0(0)$ & $1(50)$ & $0(0)$ & $1(50)$ & $0(0)$ & $1(50.0)$ & $2(100)$ & $0(0)$ & $0(0)$ \\
\hline Total $(n=90)$ & 12 (13.3) & $32(35.6)$ & $1(1.1)$ & 51 (56.7) & $1(1.1)$ & $56(62.2)$ & $64(71.1)$ & $2(2.2)$ & $10(11.1)$ \\
\hline
\end{tabular}

${ }^{a}$ No Salmonella serovars were resistant to: ceftriaxone, sparfloxacin, ciprofloxacin and moxifloxacin.

$C=$ chloramphenicol, $N A=$ nalidixic acid, $N X=$ norfloxacin, $A S=$ ampicillin, $S=$ streptomycin, $G=$ gentamicin, $C O=c o-t r i m o x a z o l e, C F X=c e f i x i m e, A K=$ amikacin $n=$ number of isolates.

Therefore the use of at least one selective enrichment broth is always highly recommended [21].

The age group 11-20 years were the most infected age group with Salmonella (35\%). These results are in agreement with Itah and Uweh in Nigeria who found that age group 11-20 years was the most infected age group (33\%) by Salmonella infection [22]. However, our results do not concur with those of Saha et al. in India who found that the age group 2-3 years was the most susceptible to Salmonella infection (35\%) [23]. Members of the age group 11-20 years are generally schoolchildren and university students who start to leave the house and eat outside the home, especially from vendors in the streets. They are more exposed to the consumption of all kinds of food and therefore more at risk as vendors may be carriers of Salmonella or their level of hygiene in the preparation of food may be very low. In general, the standard of hygiene, quality control and monitoring procedures for food preparation and storage in Thamar city is low [personal observation]. Furthermore, the people who are involved in food preparation are not very well educated or aware of the role of foods transferring pathogenic infection to the consumers.

We found that Salmonella serovars isolates were highly resistant to co-trimoxazole (71.1\%), gentamicin (62.2\%) and ampicillin (56.6\%). This finding is consistent with results of Guatam et al. who reported the high resistance of Salmonella isolates to: resistant to cotrimoxazole (74\%), gentamicin (68\%) and to ampicillin (50\%) [24]. This resistance may be due to the fact that antibiotics, particularly ampicillin and co-trimoxazole, are frequently used for treating children for almost all respiratory tract infections (viral or bacterial) and diarrhoea in almost all the private clinics and hospitals in Yemen. Indiscriminate use of these antibiotics, as it is currently practised in most of our polyclinics and private hospitals, may have led to this resistance problem. Use of antimicrobial drugs in animals feed may also lead to resistant strains of pathogens, which may be transmitted to humans through food [25]. All Salmonella isolates in our study were susceptible to ciprofloxacin, ceftriaxone, sparfloxacin and moxifloxacin. Ciprofloxacin is a currently recommended drug for the treatment of Salmonella Typhi and invasive non-typhoidal Salmonella spp. and for prophylaxis in recurrent or relapsing cases of salmonellosis [26].

Although Salmonella has been known to cause illness for over 100 years, yet it remains a public health concern in developed and developing countries, despite controls and therapeutic procedures that are already in place. The customs, traditions and sanitary level of people play an important role in controlling the spread of Salmonella, and improvement in hygiene standards will reduce the number of Salmonella infections. However, two major problems exist with regard to Salmonella infection that add to the complexity of this subject; the emergence of new serotypes and new strains resistant to the antibiotics currently available.

\section{References}

1. Xinguang $\mathrm{C}$ et al. Adults' perceived prevalence of enteric fever predicts laboratory-validated incidence of typhoid fever in children. Journal of Health, Population and Nutrition, 2007, 25:469-478.

2. Panhotra BR et al. Emerging nalidixic acid and ciprofloxacin resistance in non-typhoidal Salmonella isolated from patients having acute diarrhoeal disease. Annals of Saudi Medicine, 2004, 24:332-333.
3. Patrick ME et al. Salmonella enteritidis infection, United States, 1985-1999. Emerging Infectious Diseases, 2004, 10(1):1-7.

4. Callaway TR et al. Gastrointestinal microbial ecology and the safety of our food supply as related to Salmonella. Journal of Animal Science, 2000, 85:457-490.

5. Rustici MC et al. Antibiotic resistance among Salmonella enterica isolates. European Journal of Pediatrics, 2006, 165:577-578. 
6. Khanal B et al. Antimicrobial susceptibility patterns of Salmonella enterica serotype Typhi in Eastern Nepal. Journal of Health, Population, and Nutrition, 2007, 25:82-87.

7. Kalu IG et al. Pattern of multi-drug resistant Salmonella enterica serovar Typhi isolates in Nigeria. African Journal of Pediatrics, 2008, 7:3817-3820.

8. Holt JG et al. Bergey's manual of determinative bacteriology. Philadelphia, Lippincott, Williams \& Wilkins, 1994:215-216.

9. Grimont PAD, Weill F-X. Antigenic formulae of the Salmonella serovars, 9th ed. Paris, Institut Pasteur and World Health Organization Collaborating Centre for Reference and Research on Salmonella, 2007.

10. Rice DH et al. Household contamination with Salmonella enterica. Emerging. Infectious Diseases, 2003, 9:120-122.

11. Vollaard MA et al. Risk factors for typhoid and paratyphoid fever in Jakarta, Indonesia. Journal of the American Medical Association, 2004, 291:2607-2615.

12. Marcus R et al. Re-assessment of risk factors for sporadic Salmonella serotype Enteritidis infections: a case-control study in five FoodNet Sites, 2002-2003. Epidemiology and Infection, 2006, 135:84-92.

13. OdhahMNA. [Isolation of Salmonella species from poultryslaughter house in Thamar province] [MSc thesis]. Thamar,Yemen, Faculty of Agriculture and Veterinary Medicine, Thamar University,2009 [In Arabic].

14. Capoor MR et al. In vitro activity of azithromycin, newer quinolones and cephalosporins in ciprofloxacin-resistant Salmonella causing enteric fever. Journal of Medical Microbiology, 2007, 56:1490-1494.

15. Srikantiah P et al. Population based surveillance of typhoid fever in Egypt. American Journal of Tropical Medicine and Hygiene, 2006, 74:114-119.

16. Yousefi MR, Moshtaghi AA. Frequency of typhoidal and nontyphoidalSalmonella species and detection of their drugs resistance patterns. Journal of Research in Health Sciences, 2007, 7:49-56.

17. Cheong HJ et al. Characteristics of non-typhoidal Salmonella isolates from human and broiler- chickens in South western
Seoul, Korea. Journal of Korean Medical Science, 2007, 22:773778

18. Banajeh S. M et al. Bacterial aetiology and anti-microbial ref sistance of childhood diarrhoea in Yemen. Journal of Tropical Pediatrics, 2001, 47:301-303.

19. Alzamil FA, Alanazi AR. Serogroups and antimicrobial susceptibility of non-typhoidal salmonellas in children. Saudi Medical Journal, 2001, 22:129-132.

20. Abuxapqui JJ et al. Comparación entre medios de cultivo de selenito de sodio y tetrationato de sodio, ambos incubados a $37^{\circ} \mathrm{C}$ y $42^{\circ} \mathrm{C}$ para el aislamiento de Salmonella spp de las heces fecales de portadores [Comparison between sodium selenite and sodium tetrathionate broths, incubated at $37{ }^{\circ} \mathrm{C}$ and 42 ${ }^{\circ} \mathrm{C}$ for the isolation of Salmonella spp. from faeces of carriers]. Revista Biomédica, 2003, 14:215-220.

21. Nye KJ et al. An evaluation of the performance of XLD, DCA, $M L C B$, and $A B C$ agars as direct plating media for the isolation of Salmonella enterica from faeces. Journal of Clinical Pathology, 2002, 55:286-288.

22. Itah AY, Uweh EE. Bacteria isolated from blood, stool and urine of typhoid patients in a developing country. Southeast Asian Journal Tropical Medicine and Public Health, 2005, 36:673-676.

23. Saha MR et al. A note on incidence of typhoid Fever in diverse age group in Kolkata, India. Japanese Journal of Infectious Diseases, 2003, 56:121-122.

24. Gautam V et al. Sensitivity pattern of Salmonella serotypes in Northern India. Brazilian Journal of Infectious Diseases, 2002, 6:281-287.

25. Davis MA et al. Changes in antimicrobial resistance among Salmonella enterica serovar Typhimurium isolates from humans and cattle in the north-western United States, 1982-1997. Emerging Infectious Diseases, 1999, 5:802-804.

26. Li W et al. Ceftriaxone resistance of nontyphoidal Salmonella enterica isolates in Northern Taiwan attributable to production of CTX-M-14 and CMY-2-Lactamases. Journal of Clinical Microbiology, 2005, 43:3237-3243. 